\title{
Surgical/Theatre
}

\section{Why checklists are the RVN's best friend}

\section{Alison Young}

\section{HISTORY OF CHECKLISTS}

Safety checklists were originally introduced in the aviation industry to reduce the incidence of human error leading to a catastrophic event. They are a list of steps that must always be completed prior to any flight or movement of the aircraft, which avoids complacency and any reliance on an individual's memory. Communciation is another key area that airlines have worked hard on. There are a number of 'rules', such as non-essential conversation not being allowed below $10,000 \mathrm{ft}$ and all members of the flight crew addressing each other by first name only, removing any perceived hierachy.

\section{MEDICAL CHECKLISTS}

The World Health Organization guidelines were introduced in 2008 after the Safe Surgery Saves Lives campaign. Dr Attul Gwande had a key role in developing the checklist after observing the improvements seen in the aviation industry. There was a reduction in patient mortality after their introduction.

In the UK, the surgical saftey checklist was designed to improve communication and teamwork by bringing together the surgeons, anaesthesia providers and nurses involved in care to confrm that critical safety measures are performed during and after an operation.

Veterinary medicine, over the last few years, has developed the use of checklists within daily routines Currently they are commonly related to anaesthesia and surgery but can be created for any specific area within the practice where a need is identified. Some key organizations within the veterinary field have taken this further. The Association of Veterinary Anaesthetists has develped an anaesthesia-specific checklist that is free and downloadable. The Royal College of Veterinary Surgeons has included this in their requirements for the Practice Standards Scheme.

The evidence to show the positive results of using checklists in veterinary medicine is starting to emerge. A prospective clinical trial in Sweden concluded that the frequency and severity of postoperative complications was significantly decreased after introduction of a surgical checklist

\section{IMPLEMENTATION IN PRACTICE}

Implementing any change can be challenging, and introducing a checklist, even if the studies show the potential benefits to the patients, is not always easy. Although it may not be practical to have the whole team involved in the initial creation of the checklist, it is very important that everyone is given the opportunity to use it and provide feedback that is acted upon. Trials should be used initially until a final document that suits the needs of the practice and the people completing the form has been developed It is also important to revisit the form regularly to ensure that it remains relevant and is being completed. Audits to monitor levels of completion are also required and there should be a plan of action if this rate is low.

\section{KEY LEARNING OBJECTIVES}

- Understand the need for checklists within veterinary medicine

- Develop and implement a checklist relevant to your practice

- Identify and analyse any issues arising from implementing a checklist

\section{MULTIPLE CHOICE QUESTIONS}

1. What are the three key sections of a surgical safety checklist?

(A) Sign in, time out and sign out

(B) Preoperative, perioperative and postoperative

(C) Admit, hospitalization and discharge

(D) Log in, time out and log out

2. Why is it important to include 'potential critical events' in the time out?

(A) To plan a postoperative recovery plan for the patient

(B) To allow the team to be fully prepared for such an event

(C) To alert the owners to these potential risks

(D) To make sure the surgeon is not interrupted during the procedure

3. Whose responsibility is it to ensure the checklist is completed?
(A) The veterinary surgeon
(B) The practice manager
(C) Everyone
(D) The head nurse 